\title{
Paraplegia caused by spinal epidural abscess
}

\author{
G.C. Stephanides and R.M. Gibson \\ Department of Neurosurgery, Leeds General Infirmary, Great George Street, Leeds LS1 3EX, UK.
}

\begin{abstract}
Summary: Two patients with spinal epidural abscess, but with different neurological outcomes, are presented. The pathogenesis, clinical features and current treatment are reviewed. The need for early diagnosis is once again stressed.
\end{abstract}

\section{Introduction}

Spinal epidural abscess is an uncommon condition. The incidence is about one patient per year per large centre and any age may be affected. ${ }^{1}$ It should be considered in the differential diagnosis of a febrile patient with backache and localized tenderness. Failure to do so may have devastating consequences as we illustrate in our first case report. It is for this reason that spinal epidural abscess requires periodic review to remind clinicians of its existence as it is eminently curable if recognized early.

\section{Case reports}

\section{Case 1}

A 73 year old man, who was not diabetic, was seen in October 1986 complaining of abdominal pain radiating to his back. The patient had been discharged from hospital 8 weeks previously having been treated for Escherichia coli pneumonia. Examination revealed tenderness to percussion of the lower back but no other significant features. Radiographs were taken of the pelvis and spine and barium meal, barium enema, abdominal ultrasound and endoscopy performed. These investigations revealed the presence of a hiatus hernia but were otherwise normal. Two weeks later he was readmitted to hospital complaining of severe unrelenting backache. On examination he was pyrexial $\left(38.6^{\circ} \mathrm{C}\right)$ and had a marked cellulitis of the left leg. He had no neurological deficit. His white cell count was

Correspondence: R.M. Gibson M.D., F.R.C.S.

Accepted: 24 March 1988
$16.8 \times 10^{9} / 1$. He was commenced on oral erythromycin, cloxacillin and metronidazole and prescribed morphine intramuscularly for his backache. The antibiotic regimen was subsequently changed to oral cephalexin, clindamycin and metronidazole as his cellulitis did not respond. Three weeks after admission he complained of inability to move either leg. He was transferred to the regional neurosurgical centre where he gave a history of gradual loss of ? power in the legs over the previous 2 days. On examination he had a pronounced thoracic kyphus. Power was grade $0 / 5$ in the legs and normal in the arms. He was in urinary retention. Radiographs showed bone destruction at T11 and T12, a large right paravertebral mass and complete myelographic block at T12 (Figure 1). A provisional diagnosis of spinal epidural abscess was made. Laminectomy from $\mathrm{T} 10$ to $\mathrm{Ll}$ inclusive was performed with evacuation of the extradural pus. The wound was closed over drains and antibiotics were continued. Postoperatively no neurological improvement occurred. The sepsis in the left leg deteriorated to the extent that it required an above knee amputation. A coliform species was isolated from pus obtained from his back and also from the leg.

\section{Case 2}

A 49 year old woman, who one year previously had undergone renal transplantation and was taking prednisolone $22.5 \mathrm{mg}$ per day and azathioprine $125 \mathrm{mg}$ per day, presented to the transplant team complaining of persistent low backache of one week's duration. She was recovering from a streptococcal urinary tract infection for which she was taking oral cephalexin. Initial examination showed her to

(C) The Fellowship of Postgraduate Medicine 1988 


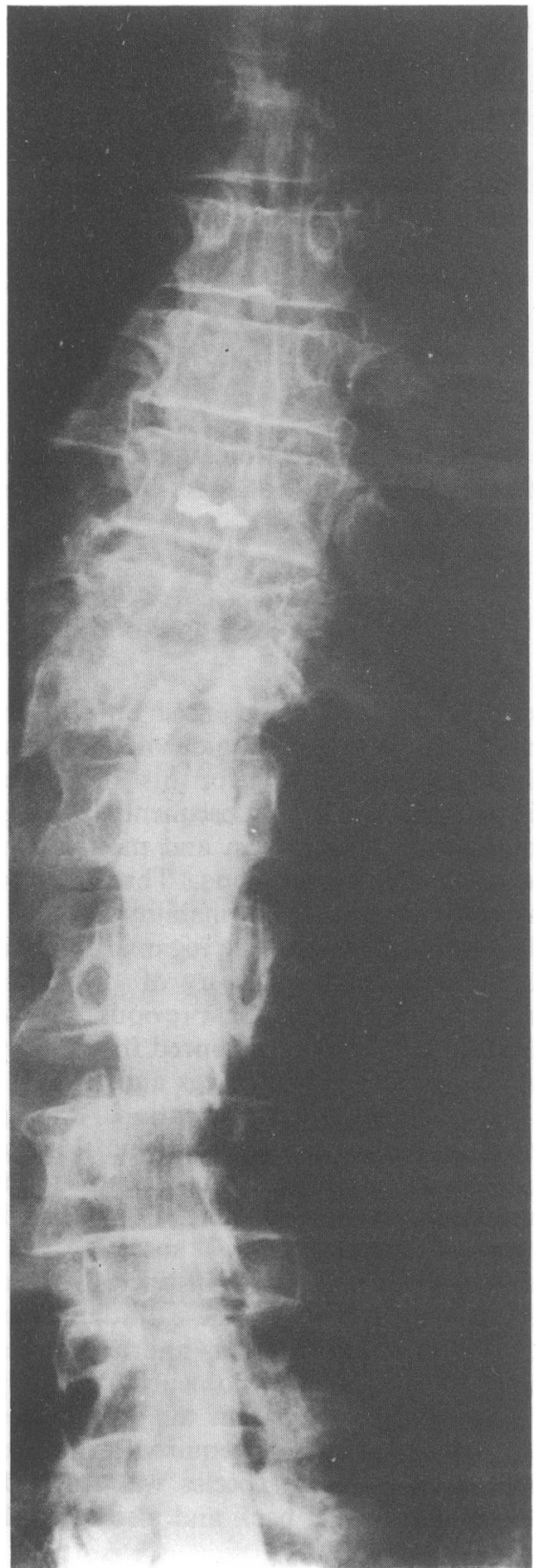

Figure 1 Case 1. Shows bone destruction at T11 and T12, large right paravertebral mass and complete myelographic block at T12.

be apyrexial but to have decreased power and sensation to pin prick in the legs. Neither the knee nor the ankle reflexes could be elicitated. Her white cell count was not raised.

The following day her temperature rose to $38.0^{\circ} \mathrm{C}$ and ampicillin was added to the antibiotic regimen.
Further examination revealed the presence of a warm swelling over the lumbosacral spine which was tender to palpation. Muscle power was $1 / 5$ in the quadriceps, $3 / 5$ in the hamstrings and $4 / 5$ below the knee bilaterally. There was decreased sensation to pin prick below the level of L4. Plain radiographs showed loss of the L4/5 interspace but were otherwise normal. The patient was transferred to the regional neurosurgical unit where myelography showed a complete block at L3/4.

Laminectomy from L3 to S1 was performed and epidural pus was found and evacuated. The wound was closed over drains and lincomycin three times a day was added to the antibiotic treatment which was continued for a total of 6 months. The patient was mobilized 3 months after the operation and at the time of her discharge she was capable of walking unaided.

\section{Discussion}

The earliest pathological description of spinal epidural abscess was given by Morgagni in 1583. The first comprehensive review was by Dandy in $1926 .{ }^{2}$

Infection in the epidural space is either blood borne, often from such unlikely sites as furuncles and infected acne, as well as cellulitis, pneumonia and urinary infection or by direct extension from vertebral osteomyelitis or neighbouring perinephric, retropharyngeal or psoas abscess. A small number of cases are iatrogenic following surgery or epidural anaesthesia. ${ }^{3}$ The commonest organism found is Staphylococcus aureus but other organisms including fungi have been described.

The presentation is almost always with pyrexia, localized tenderness and backache, which may be severe and intractable. It may also present as root pain mimicking acute abdominal conditions and/or paraparesis which rapidly progresses to paraplegia. In the early stages, prior to the development of neurological features, the white cell count and the erythrocyte sedimentation rate are generally elevated. Spinal radiographs are often normal and positive blood cultures are found in less than half the cases. Cerebrospinal fluid findings are those of parameningeal infection with white cell counts ranging from 0-800 cells $/ \mathrm{ml}$ (polymorphs and lymphocytes equally represented) with raised protein and normal glucose. ${ }^{1}$

To confirm the diagnosis, myelography, preferrably in combination with computed tomographic (CT) scanning, remains the investigation of choice. At the present time controversy surrounds the sole use of CT scanning with one report showing it to be as accurate as myelography ${ }^{4}$ and another not as useful. ${ }^{5}$ Magnetic resonance imaging has also been 
used $^{5}$ but again it was judged not to be as accurate as myelography.

Urgent surgery in the form of decompressive laminectomy with pus evacuation and equally important parenterally administered antibiotics for a minimum of 3-4 weeks (6-8 weeks if vertebral osteomyelitis is also present) remain the cornerstone of treatment. Computerized tomography guided needle aspiration of the pus has been reported as obtaining results as good as surgery in selected cases. ${ }^{4}$ Thus the role of surgery in its present form needs to be re-evaluated and reassessed, in the light of new techniques.

Several pathophysiological mechanisms have been proposed to explain the neurological deficit associated with spinal epidural abscess. These include thrombosis of the major spinal arteries and veins, impairment of the intrinsic circulation of the cord, inflammatory vasculitis leading to vascular com-

\section{References}

1. Baker, A.S., Ojeman, R.G., Swartz, M.N. \& Richardson, E.P. Spinal epidural abscess. N Engl J Med 1975, 293: $463-468$.

2. Dandy, W.E. Abscesses and inflammatory tumours in the spinal epidural space (so-called pachymeningitis externa). Arch Surg 1926, 13: 477-494.

3. Loarie, D.J. \& Fairley, H.B. Epidural abscess following spinal anaesthesia. Anesth Analg 1978, 57: 351-353.

4. Leys, D., Lesoin, F., Viaud, C. et al. Decreased morbidity from acute bacterial spinal epidural abscess using computed tomography and nonsurgical treatment in selected patients Ann Neurol 1985, 17: 350-355.

5. Chappel, R., Verhelst, J.A., Nagler, J.M., Dom, L., Appel, B. \& Herregods, P. Epidural abscess causing tetraparesis: case report. Paraplegia 1986, 24: 364-369. promise and mechanical cord compression. ${ }^{6-8}$ Recent experiments using an animal model in which the clinical, radiological and bacteriological aspects of spinal epidural abscess in humans were reproduced, demonstrated histopathological evidence consistent with mechanical obstruction and pressure necrosis. ${ }^{9}$

The prognosis of the disease is related to the degree of neurological impairment and particularly to the length of time it has been present when definitive treatment is undertaken, as exemplified by the two cases presented. In a series of 18 cases, none of the 5 cases with paraplegia of over 48 hours duration improved. ${ }^{10}$

Spinal epidural abscess is an uncommon but serious infection. The finding of spinal ache, localized tenderness and fever should alert one to the diagnosis and warrants urgent investigation, ideally prior to the development of neurological deficit.

6. Russel, N.A., Vaughan, R. \& Morley, T.P. Spinal epidural infection. Can J Neurol Sci 1979, 6, 325-328.

7. Browder, J. \& Meyers, R. Infections of the spinal . epidural space: an aspect of vertebral osteomyelitis. Am J Surg 1937, 37: 4-26.

8. Browder, J. \& Meyers, R. Pyogenic infections of the spinal epidural space: a consideration of the anatomic and physiologic pathology Surgery 1941, 10: 296-308.

9. Feldenzer, J.A., McKeever, P.E., Schaberg, D.R., Campbell, J.A. \& Hoff, J.T. Experimental spinal epidural abscess: a pathophysiological model in the rabbit. Neurosurgery 1987, 20: 859-867.

10. Yang, S. Spinal epidural abscess. $N Z$ Med J 1982 , 95: 302-303. 